\title{
Hausdorff dimension and hierarchical system dynamics
}

\author{
K. Lukierska-Walasek* \\ Institute of Physics \\ University of Zielona Góra \\ ul. Z. Szafrana $4 \mathrm{a}$ \\ 65-516 Zielona Góra, Poland
}

\author{
K. Topolski ${ }^{\dagger}$ \\ Institute of Mathematics, \\ Wrocław University, \\ Pl. Grunwaldzki 2/4, \\ 50-384 Wrocław, Poland
}

\begin{abstract}
We show that Hausdorff dimension may be used to distinguish different dynamics of the relaxation in hierarchical systems. We examine the hierarchical systems following the temperature-dependent power-law decay and the Kohlrausch law. For our purposes, we consider a Lévy stochastic processes on $p$-adic integer numbers.
\end{abstract}

\section{Introduction}

The Lévy stochastic processes are natural generalization of the Brownian motion [11, 19]. The foundation for this generalization is the theory of the infinitely divisible probability distributions established by by the works of Khintchine [14, 15] and Lévy [11. The further development is presented in the book of Gniedenko and Kolmogorov [12. Lévy processes are widely used to describe and model a wide range of physical processes, such as turbulence [16, chaotic dynamics [30, plasma physics 31] and phase transition [8]. Let us notice that the Lévy processes are frequently appear in financial dynamics $[23$, biology [28].

In the case of the Euclidean space the $\alpha$-stable Lévy processes are characterized by the Lévy index $0<\alpha \leq 2$, and when $\alpha=2$ we obtain the Gaussian process. The transition probabilities of the $\alpha$-stable Lévy process are solution of the fractional diffusion equation with fractional Riesz derivative [26]. The fractal dimension of stable Lévy process with index $\alpha \in(0,1]$ is equal $\alpha$ [7]. In the case of the $\mathbb{Q}_{p}$ space the similar results for the $\alpha$-stable Lévy processes hold [17, 9 .

In our paper we consider the Lévy process in the space of $p$-adic numbers. We consider the Hausdorff dimension of the spherically symmetric Lévy processes which are used to describe the relaxation in hierarchical systems. We study the physical system which evolves in time by a jump from state to state in hierarchical space. Hierarchical structure of metastable states corresponds to such structure are expressed in terms of pure states in the sense of Prisi solution [22. The transitions between states are thermally activated with rates determined by the free energy barriers separating the states. The simplest case is a system with linearly growing, with distance, set of barriers. For such system we have the temperature-dependent power law of relaxation dynamics. Another case is a system with barriers grow in slower way, for example proportional to logarithm of distance separated states. In this case the system dynamics fulfills, for large $t$, the Kohlrausch law 24,20 .

To distinguish system with different dynamics we compare the Hausdorff dimension of the trajectory of the processes describing relaxation.

*e-mail: klukie@proton.if.uz.zgora.pl

†e-mail: topolski@math.uni.wroc.pl 


\section{Lévy processes with spherically symmetric measure}

Let $p$ be an arbitrary prime number and let $\mathbb{Q}_{p}$ denote the set of $p$-adic numbers. A $p$-adic number is a formal series, $\sum_{i \geq-M} a_{i} p^{i}$, with coefficients $a_{i}$ satisfying $0 \leq a_{i} \leq p-1$, where $M<\infty$. With this definition, a $p$-adic number $a=\sum_{i \geq-M} a_{i} p^{i}$ can be identified with the sequence $\left(a_{i}\right)_{i \geq-M}$ of its coefficients.

In order to introduce a distance between $p$-adic numbers $a$ and $b$ let us first consider an order of a $p$-adic number. The order of a $p$-adic number $a=\left(a_{i}\right)_{i \geq-M}$ is the smallest $m$ for which $a_{m} \neq 0$

$$
\operatorname{ord}_{p}(a)=\min \left\{i: a_{i} \neq 0\right\},
$$

with the convention that minimum of the empty set is equal infinity.

Now, in term of the function $\operatorname{ord}_{p}(\cdot)$, we may introduce in the space of $p$-adic number the norm $\|\cdot\|_{p}$

$$
\|x\|_{p}=p^{-\operatorname{ord}_{p}(x)}
$$

and the $p$-adic metrics

$$
d_{p}(x, y)=\|x-y\|_{p}=p^{-\operatorname{ord}_{p}(x-y)}
$$

For any $x_{0} \in \mathbb{Q}_{p}$ and any integer number $r \in \mathbb{Z}$ we may define a closed $p$-adic ball $K\left(a, p^{r}\right)$ with center $a$ and radius $p^{r}$

$$
K\left(x_{0}, p^{r}\right)=\left\{x \in \mathbb{Q}_{p},\left\|x-x_{0}\right\|_{p} \leq p^{r}\right\} .
$$

The family of all such balls constitutes a countable topological base for $\mathbb{Q}_{p}$. By $d x$ we denote the normalized Haar measure, $\int_{\|x\|_{p} \leq 1} d x=1$, on Borel $\sigma$-field $\mathcal{B}$ of subsets of the space $\mathbb{Q}_{p}$. Let $\chi(a)$ denotes a normalized additive character of $\mathbb{Q}_{p}$, defined by the formula

$$
\chi(a)=\exp (2 \pi i\{a\})
$$

where $\{a\}$ is the fractional part of $a \in \mathbb{Q}_{p}$.

The Fourier transform of a complex valued function $f \in L_{1}\left(\mathbb{Q}_{p}\right)$ is defined for $a \in \mathbb{Q}_{p}$ by

$$
\hat{f}(a)=\int_{\mathbb{Q}_{p}} \chi(a x) F(x) d x,
$$

We consider the Lévy process on $\mathbb{Q}_{p}$, defined as in 9 ]

Definition 1 The Hunt process $X=\left(\Omega, \mathcal{F}, \mathcal{F}_{t}, X_{t}, \theta_{t}, P_{x}\right)$ with state space $\mathbb{Q}_{p}$ and adjoined terminal state $\Delta$ is a Lévy process on $\mathbb{Q}_{p}$ if

(1) for $t \geq 0$ and $A$ a Borel subset of $\mathbb{Q}_{p} P_{x}\left(X_{t} \in A\right)=P_{0}\left(X_{t}+x \in A\right)$,

(2) for all $t \geq 0 P_{0}\left(X_{t} \in \mathbb{Q}_{p}\right)=1$.

Let $X$ be a Lévy process on $\mathbb{Q}_{p}$ with transition function $F_{t}(d x)$. The Fourier transform of the transition function of the process $X$

$$
\hat{\Phi}(t, a)=\int_{\mathbb{Q}_{p}} \chi(x a) F_{t}(d x),
$$

has the following Lévy-Khinchine representation

$$
\hat{\Phi}(t, a)=\exp \left\{t \int_{\mathbb{Q}_{p}}(\chi(x a)-1) \nu(d x)\right\},
$$


where $t \in \mathbb{R}$ and $\nu$ is Lévy measure of the Lévy process which satisfies for all $n \in \mathbb{N}$ condition

$$
\nu\left(\mathbb{Q}_{p} \backslash\left\{\|x\|_{p} \leq p^{n}\right\}\right)<\infty .
$$

We now turn to the consideration of special class of Lévy processes on $\mathbb{Q}_{p}$ defined in the following way.

Let $\{a(M), M \in \mathbb{Z}\}$ be a sequence of real numbers satisfying the following two conditions

(i) $a(M) \geq a(M+1)$,

(ii) $\lim _{n \rightarrow \infty} a(n)=0$.

The $\sigma$-finite measure $\nu$ on $\mathbb{Q}_{p}$ defined by

$$
\nu(d x)=\sum_{M=-\infty}^{\infty} \frac{a(M)-a(M+1)}{p^{M}(p-1)} \mathbb{I}_{\left\{\|x\|_{p}=p^{M+1}\right\}}(x) d x
$$

is a spherically symmetric Lévy measure.

The transition probability of a spherically symmetric Lévy process can be written explicitly $[3]$. Denote

$$
P_{M}=\left(1-p^{-1}\right) \sum_{i=0}^{\infty} p^{-i} \exp \left\{t(1-p)^{-1}(p a(M+i)-a(M+i+1))\right\},
$$

then the transition probability $P_{t}$ of the process with Lévy measure (1) is given by

$$
P_{t}\left(x_{0}, K\left(x, p^{M}\right)\right)=P_{M}(t)
$$

if $\left\|x_{0}-x\right\| \leq p^{M}$, and by

$$
P_{t}\left(x_{0}, K\left(x, p^{M}\right)\right)=p^{1-m}(p-1)^{-1}\left(P_{M+m}(t)-P_{M+m-1}(t)\right),
$$

if $\left\|x_{0}-x\right\|=p^{M+m}, m \geq 1$.

The Lévy processes with spherically symmetric Lévy measures are examples of Markov processes with $\mathbb{Q}_{p}$ as the state space considered by Albeverio and Karwowski [1, 2]. The spherically symmetric Lévy processes have been studied by probabilistic approach by many authors 19, 1, 2, 29, 13, they were used to study the hierarchical systems dynamics in [5, 6, 20] The analytical approach to examine spherically symmetric Lévy processes on $\mathbb{Q}_{p}$ was presented in [4, 5, 6, 18, 17]. In this approach authors use the pseudo-differential operators. The simplest of them, the fractional differentiation operator $D^{\alpha} \alpha>0$, was introduced by Vladimirov and is defined by [27]

$$
D^{\alpha} f(x)=\frac{1-p^{\alpha}}{1-p^{-\alpha-1}} \int_{\mathbb{Q}_{p}}\|y\|_{p}^{-\alpha-1}(f(x-y)-f(x)) d y,
$$

where $f \in D\left(\mathbb{Q}_{p}\right)$, the space of locally constant function with compact supports. The transition probabilities of $\alpha$-stable Lévy process on $\mathbb{Q}_{p}$ are solution of the following Cauchy problem

$$
\frac{\partial}{\partial t} f(x, t)+a\left(D^{\alpha} f\right)(x, t)=0,
$$

where $f(x, 0)=\delta(x)$ and $a>0[17$. 


\section{$3 \quad$ Hausdorff dimension of Lévy processes on $p$-adic integers.}

In this section we first briefly recall the definition of some basic notions about Hausdorff dimension. Then we will describe Hausdorff dimension for processes studied in this paper. For the general theory of Hausdorff measure and Hausdorff dimension we refer to [25, 10, 21]. The Hausdorff measure is based on the notation of a covering of the metric space $E$ by sets of finite diameter. A covering of $E$ is an at most countable collection of sets $E_{1}, E_{2}, \ldots$ with

$$
E \subseteq \bigcup_{i=1}^{\infty} E_{i}
$$

For every $s \leq 0$ we say that the $s$-value of the covering is $\sum_{i=1}^{\infty}\left|E_{i}\right|^{s}$, where $\left|E_{i}\right|$ denotes the diameter of the set $E_{i}$.

Definition 2 For every $\alpha \geq 0$ the $\alpha$-Hausdorff measure of a subset $A \subset E$ of a metric space $E$ is defined as

$$
\mathcal{H}^{\alpha}(A)=\lim _{\delta \downarrow 0} \mathcal{H}_{\delta}^{\alpha}(A)
$$

where

$$
\mathcal{H}_{\delta}^{\alpha}(A)=\left\{\inf \left\{\sum_{i=1}^{\infty}\left|E_{i}\right|^{\alpha}:\left(E_{i} ; i \in N\right) \text { is a covering of } A \text { and }\left|E_{i}\right|<\delta\right\} .\right.
$$

It can be shown that $\mathcal{H}^{\alpha}$ is a measure on Borel subsets of $E$ and if $0 \leq s<t<\infty$ and $A \subset E$ then

(1) $\mathcal{H}^{s}(A)<\infty$ implies $\mathcal{H}^{t}(A)=0$,

(2) $\mathcal{H}^{t}(A)>0$ implies $\mathcal{H}^{s}(A)=\infty$.

Thus we can defined Hausdorff dimension in the following way.

Definition 3 The Hausdorff dimension of a subset $A \operatorname{dim}(A)$ is defined as

$$
\begin{aligned}
\operatorname{dim} A & =\inf \left\{s: \mathcal{H}^{s}(A)=0\right\}=\inf \left\{s: \mathcal{H}^{s}(A)<\infty\right\} \\
& =\sup \left\{t: \mathcal{H}^{t}(A)>0\right\}=\sup \left\{t: \mathcal{H}^{t}(A)=\infty\right\} .
\end{aligned}
$$

In order to study sample path properties of the Lévy processes on the space of $p$-adic integers, we can use the results by Evans, who investigated Lévy processes on a locally compact totally disconnected Abelian topological group. As in a case of Euclidean Lévy process Hausdorff dimension of the path of $p$-adic Lévy process, $\{X(t) ; t \geq 0\}$, is characterized in terms of exit times.

Let for $n \geq 0$

$$
\tau(n)=\inf \left\{t \geq 0: X(t) \notin K\left(0, p^{-n}\right)\right\}
$$

with the convention that $\inf \emptyset=\infty$, and denote

$$
q(n ; N)=P\left\{X(t) \notin K\left(0, p^{-n}\right), \text { for all } t \in[\tau(n), \tau(N))\right\} .
$$

For any Borel set $B \subset \mathbb{R}_{+}$we define the range or path to be the random set generated by $X$

$$
X(B)=\left\{x \in \mathbb{Q}_{p}: x=X(t) \text { for some } t \in B\right\} .
$$

The Hausdorff dimension of the sample path of spherically symmetric Lévy processes on $p$-adic integers is described in the following result (see ([9] for a proof). 
Theorem 1 Suppose that the exists an integer $N$ such that $\nu\left(\mathbb{Z}_{p} \backslash K\left(p^{-N}\right)>0\right.$, and for all $\rho>0$

$$
\lim _{n \rightarrow \infty} p^{n \rho} q(n ; N)=\infty,
$$

then

$$
P\left(\beta^{\prime} \operatorname{dim} B \leq \operatorname{dim} X(B) \leq \beta^{\prime \prime} \operatorname{dim} B \text { for all } B\right)=1,
$$

where

$$
\begin{aligned}
\beta^{\prime} & =\inf \left\{\beta: \liminf _{n \rightarrow \infty} p^{-\beta n} q(n ; N) \nu\left(\mathbb{Z}_{p} \backslash K\left(0, p^{-n}\right)\right)=0\right\}, \\
\beta^{\prime \prime} & =\inf \left\{\beta: \limsup _{n \rightarrow \infty} p^{-\beta n} q(n ; N) \nu\left(\mathbb{Z}_{p} \backslash K\left(0, p^{-n}\right)\right)=0\right\} .
\end{aligned}
$$

Let $\{X(t): t \geq 0\}$ be the Lévy processes on $p$-adic integers with Lévy measure $\nu$ such that for all $n \geq 1$ and for some $\alpha \in(0,1)$

$$
\nu\left(\mathbb{Z}_{p} \backslash K\left(0, p^{-n}\right)\right)=p^{-\alpha n},
$$

then it was shown by Evans ([9], page 253) that

$$
P(\operatorname{dim} X(B)=\alpha \operatorname{dim}(B) \text { for all } B)=1 .
$$

In the similar way we can show that for a Lévy processes $\{X(t): t \geq 0\}$, on $p$-adic integers with Lévy measure $\nu$ such that for all $n \geq 1$ and for some $\alpha>0$

$$
\nu\left(\mathbb{Z}_{p} \backslash K\left(0, p^{-n}\right)\right)=p^{-\alpha \ln n},
$$

we have

$$
P(\operatorname{dim} X(B)=0 \text { for all } B)=1 \text {. }
$$

Now we use these results to analyze relaxation dynamics in hierarchical system. The simplest model of diffusion on hierarchical space is the model proposed by Ogielski and Stein 24. They consider a regular Cayley tree with $M$ levels and fixed branching ratio $p$. The natural ultrametric distance $d(k, l)$ between leaves $k$ and $l$, is defined as equal to the height $m, m=0,1, \ldots, M$ of their closest common ancestor. Transitions between states are thermally activated. The height of the energy barriers, $\Delta_{k} k=1,2, \ldots$, which the system overcomes can be ordered in the increasing sequence $\Delta_{1}<\Delta_{2}<\ldots<\Delta_{k} \ldots$ Now, identifying the states $x$ and $y$ separated by the energy barrier $\Delta_{m}$ with leaves $k$ and $l$, the probability of moving from state $x$ to state $y$ may be defined as equal to transition probability from leaf $k$ to leaf $l$ separated by the ultrametric distance $m$. Thus dynamics in the space of states separated by the energy barrier may be studied in terms of appropriate stochastic process involving the end points of Cayley tree, as a space of states.

The rate of transition from a starting site at the Cayley tree to another site lying at an ultrametric distance $k$ is given by $\exp \left\{-\Delta_{k} / T\right\}$, where $T$ is normalized temperature. We may represent the end points of regular Cayley tree with $M$ levels and fixed branching ratio $p$ as a set of disconnected balls with fixed radius $p^{-M}$ covering space of $p$-adic integer $\mathbb{Z}_{p}$ and define an appropriate spherically symmetric $\mathbb{Z}_{p}$-valued Lévy process $[20$.

For the sequence of barriers linearly growing with distance $\Delta_{k}=\Delta k$ for some positive constant $\Delta$ we consider $\mathbb{Z}_{p}$-valued Lévy process $\{X(t) ; t \geq 0\}$ with a Lévy measure $\nu(d x)$ such that

$$
\nu\left(\mathbb{Z}_{p} \backslash K\left(0, p^{-n}\right)\right)=p^{-\alpha n},
$$

where $\alpha=\Delta(T \ln p)^{-1}$.

If $\Delta$ and $T$ are such that $\Delta(T \ln p)^{-1}<1$, then Hausdorff dimension of the path $X([0,1])$ is equal 
to $\alpha=\Delta(T \ln p)^{-1}$, with probability one.

For the sequence of barriers growing in a slower way, $\Delta_{k}=\Delta \ln k$, we consider $\mathbb{Z}_{p}$-valued Lévy process $\{Y(t) ; t \geq 0\}$ with a Lévy measure $\nu(d x)$ such that

$$
\nu\left(\mathbb{Z}_{p} \backslash K\left(0, p^{-n}\right)\right)=p^{-\alpha \ln n},
$$

where $\alpha=\Delta(T \ln p)^{-1}$.

In this case Hausdorff dimension of the path $Y([0,1])$ of the process is equal 0 , with probability one.

\section{References}

[1] S. Albeverio, W. Karwowski, A random walk on $p$-adics - the generator and its spectrum. Stochastic. Process. Appl. 53 (1994), 1-22.

[2] S. Albeverio, W. Karwowski, X. Zhao, Asymptotics and spectral results for random walks on p-adics. Stochastic. Process. Appl. 83 (1999), 39-59.

[3] S. Albeverio, X. Zhao, A decomposition theorem for Lévy processes on local fields. J. Theor. Probab. 14, (2001), 1-19.

[4] V. A. Avetisov, A. H. Bikulov, S. V. Kozyrev, Application of $p$-adic analysis to models of breaking of replica symmetry. J. Phys. A: Math. Gen. 32 (1999), 8785-8791.

[5] V. A. Avetisov, A. H. Bikulov, S. V. Kozyrev, V. A. Osipov, p-adic models of ultrametric diffusion constrained by hierarchical energy landscapes. J. Phys. A: Math. Gen. 35 (2002), $177-189$.

[6] V. A. Avetisov, A. H. Bikulov, V. A. Osipov, p-adic description of characteristic relaxation in complex systems. J. Phys. A: Math. Gen. 36 (2003), 4239-4246.

[7] R. M. Blumenthal and R. Getoor, Some theorems on stable processes. Trans. Amer. Math. Soc. 95 (1960), 263-273.

[8] E. Dobierzewska-Mozrzymas, P. Biegański, E. Piecul and J. Wójcik, Statistical description of systems on the basis of the Mandelbrot law: discontinuous metal films on dielectric substrates. J. Phys. Condense Matter 11 (1999), 5561-5568.

[9] S. N. Evans, Local properties of Lvy processes on a totally disconnected group. J. Theoret. Probab. 2 (1989), 209-259.

[10] H. Federer, Geometric Measure Theory, Die Grundlehren der math. Wiss Band 153, SpringerVerlag, Berlin, 1969.

[11] C. W. Gardiner, Handbook of Stochastic Methods, Springer-Verlag, Berlin, 1985.

[12] B. V. Gnedenko i A. H. Kolmogorov, Limit Distributions for Sums of Independent Random Variables, (Gostekhizdat, Moscow, 1949; Addison-Wesley, Reading, MA, 1968).

[13] H. Kaneko, Time-inhomogeneous stochastic processes on the $p$-adic number field. Tohoku Math. J. 55 (2003), 65-87.

[14] A. Ya. Khintchine, Zur Theorie der unbeschränkt teilbaren Verteilungsgesetzen. Mat. Sb. 2(1) (1937), 79-119. 
[15] A. Ya. Khintchine, Limit Laws for Sums of Independent Random Varables (ONTI NKTP SSSR, Moscow, 1938)[in Russian].

[16] J. Klafter, A. Blumen and M. F. Shleisinger, Stochastic pathway to anomalous diffusion. Phys. Rev. A 35 (1987), 3081-3085.

[17] A. N. Kochubei, Pseudo-Differential Equations and Stochastic over Non-Archimedean Fields, Marcel Dekker, Inc, New York, 2001.

[18] S. V. Kozyrev, V. A. Osipov, V. A. Avetisov, Nondegenerate ultrametric diffusion. J. Math. Phys. 46 (2005), 063302-063317.

[19] P. Lévy, Théorie de l'Addition des Variables Alétores, Gauthier-Villars, Paris, 1937.

[20] K. Lukierska-Walasek and K. Topolski, Random walk on p-adics and hierarchical systems. Phys. Rev. B 73 (2006), 054419-054425.

[21] P. Mattila, Geometry of sets and measures in Euclidean spaces. Fractals and rectifiability, Cambridge University Press, Cambridge, 1999.

[22] M. Mezard, G. Parisi, A. Virasoro, Spin glass theory and beyond, World Scientific, Singapore, 1987.

[23] R. N. Montega and H. E. Stanley, Scaling behaviour in the dynamics of an economic index. Nature (London) 376 (1995), 46-49.

[24] A. T. Ogielski, L. D. Stein, Dynamics on ultrametric spaces. Phys. Rev. Lett. 55 (1985), 1634.

[25] C. A. Rogers, Hausdorff Measures, Cambridge University Press, Cambridge, 1970.

[26] K. Sato, Lévy processes and Infinitely Divisible Distributions, Cambridge Univ. Press, Cambridge, 1999.

[27] V. S. Vladimirov, I. V. Volovich, E. I. Zelenov, p-adic analysis and mathematical physics, (Nauka, Moscow, 1994; World Scientific, Singapore, 1994).

[28] B. J. West and W. Deering, Fractal physiology for physicists: Lévy statistics Phys. Rep. 246 (1994), 1-100.

[29] K. Yasuda, Additive processes on local fields. J. Math. Sci. Univ. Tokyo 3 (1996), 629-654.

[30] G. M. Zaslavsky, Fractional kinetic equation for Hamiltonian chaos. Physica D 76 (1994), $110-122$.

[31] G. Zimbardo, P. Veltri, G. Basile and S. Prinigato, Anomalous diffusion and Lévy random walk of magnetic field lines in three dimensional turbulence. Phys. Plasmas 2 (1995), 2653-2663. 